\title{
$\begin{array}{ll}\text { Research Square } & \text { Preprints are preliminary reports that have not undergone peer review. } \\ \text { They should not be considered conclusive, used to inform clinical practice, }\end{array}$ or referenced by the media as validated information.
}

\section{The Impacts of G4S Mutation on N-glycosylation and conformation of the Human Coagulation Factor IX GLA domain: In silico and In vitro Analysis}

\section{Fahimeh Ghasemi}

Birjand University of Medical Sciences https://orcid.org/0000-0002-5870-3396

Mina Maddah

KN Toosi: KN Toosi University of Technology

Hourieh Kalhor

Qom University of Medical Sciences

Mohsen Khorashadizadeh

Birjand University of Medical Sciences

Alireza Zomorodipour ( $\nabla$ zomorodi@nigeb.ac.ir)

National Institute for Genetic Engineering and Biotechnology https://orcid.org/0000-0003-0671-4779

\section{Research Article}

Keywords: Hemophilia B, Human coagulation factor IX, N-glycosylation mutation, GLA domain

Posted Date: December 3rd, 2021

DOI: https://doi.org/10.21203/rs.3.rs-1071688/v1

License: (9) This work is licensed under a Creative Commons Attribution 4.0 International License.

Read Full License 


\section{Abstract}

Missense mutations are the most prevalent form of mutation in hemophilia B patients. These alterations may result in the creation of novel and non-native N-glycosylation sites (Asn-X-Ser/Thr) through single amino acid substitutions. The pathogenic mechanisms of $\mathrm{N}$-glycosylation mutations in hemophilia $\mathrm{B}$ patients have not been extensively studied yet. By survey among known missense mutations, we found only one $\mathrm{N}$-glycosylation mutation in the $\mathrm{Y}$-carboxyglutamic-rich (GLA) domain of the human coagulation factor IX (hFIX). This mutation that was reported in patients with mild and moderate hemophilia $\mathrm{B}$, is caused by G4S amino acid substitution. To investigate the possibility of glycan attachment to the novel $\mathrm{N}$-glycosylation site in G4S-mutant hFIX and the occurrence of hyperglycosylation, site-directed mutagenesis was applied to introduce the selected mutation into the coding sequence of the hFIX. The nucleotide sequences of the both native and G4S-mutant hFIX were separately cloned into the pcDNA3.1 expression plasmid and transiently expressed in HEK293T cells. Our results from gradient SDS-PAGE and western blotting analysis of the both recombinant native and mutant hFIX demonstrated no glycan attachment to the new N-glycosylation site in the G4S-mutant hFIX. Molecular dynamics (MD) simulation was also conducted to provide atomistic insights into structure and behavior of the native and G4Smutant GLA domains in the both free and membrane-bound states. The results revealed that the mutation slightly affected the dynamic behavior of the mutant GLA domain. The conformational analysis proved that the native GLA domain had less fluctuation and more stability than the mutant GLA domain. The slight conformational changes may influence the binding capacity and interaction of the mutant GLA domain to phospholipid bilayer which is necessary for coagulation activity of the hFIX. These findings were in accordance with the nature of the G4S mutation which causes mild hemophilia B.

\section{Introduction}

Hemophilia B is a kind of recessive X-linked bleeding disorder caused by the complete absence or reduced level of the functional human coagulation factor IX (hFIX) (Bolt et al., 2012). Its incidence rate is one in 25,000 live male newborns worldwide (Zacchi et al., 2021). According to the hFIX coagulation activity (FIX:C), hemophilia $B$ is classified as severe $\left(<1 \mathrm{IU} \mathrm{dL}^{-1}\right)$, moderate $\left(1-5 \mathrm{IU} \mathrm{dL}^{-1}\right)$ and mild $(5-40$ IU dL ${ }^{-1}$ ) (Goodeve, 2015). The $\mathrm{hFIX}$ is a vitamin $\mathrm{K}$-dependent plasma serine protease that plays a vital role in the intermediate stage of the blood coagulation cascade (Bolt et al., 2012; Huang et al., 2004; Zacchi et al., 2021). The hFIX gene was first cloned in 1982-1983, and its full nucleotide sequence was published in 1985 (Goodeve, 2015; Yoshitake et al., 1985). The hFIX gene is composed of eight exons that encode a pre-pro-protein of 461 amino acids. The pre-pro-protein is subsequently processed into a 415residue mature hFIX (Goodeve, 2015; Pegg et al., 2020; Yoshitake et al., 1985).

The mature form of the hFIX consists of four distinct structural domains: a $Y$-carboxyglutamic acid-rich (GLA) domain (residues 1-46) at the N-terminus, followed by two epidermal growth factor (EGF)-like domains (EGF1 and EGF2). The GLA domain of hFIX has high affinity toward $\mathrm{Ca}^{2+}$ ions which is required for its membrane binding (Ohkubo \& Tajkhorshid, 2008). 
The GLA domain is composed of an N-terminal $\omega$-loop (residues 1-11), followed by three short a-helices: helix A (residues 14-19), helix B (residues 24-32) and helix C (residues 35-45) (Huang et al., 2004; Ohkubo \& Tajkhorshid, 2008). A 35-residue activation peptide connects the EGF2 domain to the C-terminal serine protease (SP) domain. (Gui et al., 2002).

Proteolytic cleavage of the activation peptide by either factor Xla (FXla) or factor VIla/tissue factor (FVIlaTF) complex results in the formation of the active hFIX (hFIXa) (Persson et al., 1998). The hFIXa consists of two light and heavy chains that are linked to each other by disulfide bonds. The N-terminal light chain (residues 1-145) is composed of GLA, EGF1 and EGF2 domains whereas the heavy chain (residues 181415) contains of c-terminal serine protease (SP) domain (Vadivel \& Bajaj, 2012; Venkateswarlu, 2014).

The hFIXa in association with its co-factor, factor VIIla (FVIIla), converts the factor X (FX) to its active form, FXa (Autin et al., 2005; Bolt et al., 2012; Huang et al., 2004). The FXa, in turn, catalyzes the conversion of the prothrombin to thrombin which itself subsequently converts the fibrinogen to fibrin clots (Ohkubo \& Tajkhorshid, 2008).

The hFIX is a glycoprotein with two native N-linked oligosaccharides attached to Asn ${ }^{157}$ and Asn ${ }^{167}$ residues within the activation peptide (Gil et al., 2008). N-glycosylation is a kind of post-translational modification that occurs on the majority of secretory and membrane proteins in eukaryotic cells. During $\mathrm{N}$-glycosylation process, a glycan moiety is attached to the amide side-chain of an asparagine residue placed within the consensus sequence Asn-X-Ser/Thr, where $\mathrm{X}$ can be any amino acid except proline (Bolt et al., 2012; Gil et al., 2008).

Numerous mutations, including point mutations, deletions, insertions, duplications, insertions and deletions, and complex alterations (large deletions and insertions) have been reported in the coding sequence of hFIX, which resulted in hemophilia B. It is well documented that missense mutations account for a significant portion (about $65 \%$ ) of all mutations in the hereditary disorders such as hemophilia B (Goodeve, 2015; Wei et al., 2018). Missense mutations, may result in the creation of an extra and nonnative $\mathrm{N}$-glycosylation site, through single amino acid substitution. The subsequent glycan attachment to the asparagine residue in the new $\mathrm{N}$-glycosylation site might negatively affect protein function and secretion (Vogt et al., 2005; Vogt et al., 2007). The gain-of-glycosylation mutation, which accounts for up to 1.4 percent of all disease-causing missense mutations, plays a key role in the development of various genetic disorders (Casanova \& Abel, 2007; Vogt et al., 2005; Vogt et al., 2007). In the previous study that was conducted by Vogt et al in 2005, three children were diagnosed with Mendelian Susceptibility to Mycobacterial Disease (MSMD) which was caused by a gain-of-glycosylation mutation in the interferon $Y$ receptor ligand-binding chain (IFNYR2) (Vogt et al., 2005). The homozygous children, respect to this mutation, were susceptible to mycoplasma infection (Vogt et al., 2005; Vogt et al., 2007). Moreover, in patients with hemophilia A, various missense mutations in the hFVIII coding sequence were identified which gave rise to the occupied new N-glycosylation sites (Wei et al., 2018; Wei et al., 2017). However, this kind of mutation has not been well studied in the case of the hFIX and hemophilia B patients. 
The G4S mutation, located within the N-terminus GLA domain of the hFIX, is a kind of missense mutation that causes mild hemophilia B (Saad et al., 1994; Van de Water et al., 1996). This mutation results in the creation of a new and non-native N-glycosylation site with Asn ${ }^{2}-$ Ser $^{3}-$ Ser $^{4}$ sequence (Saad et al., 1994). But it is worth pointing that mere introduction of a new $\mathrm{N}$-glycosylation site never guarantees the attachment of an oligosaccharide chain (Lowenthal et al., 2016). It is well documented that there are plenty of potential $\mathrm{N}$-glycosylation sites in the eukaryotic proteome that are not occupied with a glycan (Gil et al., 2008).

In this study, our aim was first to investigate the glycan attachment to the new N-glycosylation site in the G4S-mutant hFIX using experimental methodologies. For this purpose, we employed HEK293T cells to express recombinant native and G4S-mutant hFIX. We also used computational and bioinformatics tools to investigate the structural alterations caused by the G4S mutation in the GLA domain of the $h F I X$. Interestingly, our results from experimental analysis showed no glycan attachment to the new $\mathrm{N}$ -

glycosylation site in the G4S-mutant hFIX. Furthermore, according to the results of the molecular dynamic simulations, the native GLA domain showed less fluctuation and was more stable than the mutant GLA domain. It is suggested that the G4S mutation slightly influenced the three-dimensional (3D) structure of hFIX GLA domain. The findings were in line with the nature of the G4S mutation which causes mild hemophilia B.

\section{Materials And Methods Experimental studies}

\section{Preparation of the mutant $\mathrm{hFIX}$ sequence and construction of recombinant plasmid}

The overlap extension PCR was applied to generate the G4S-mutant hFIX. The nucleotide sequence encoding the native hFIX was first selected from GenBank (accession number: NM_000133.4). Then two pairs of primers (internal and external) were designed to introduce the G4S mutation into the hFIX coding sequence. The primers sequences are listed in Table 1. The internal primer, G4S-F and G4S-R, were designed in a way to cover the mutation site and introduce the desired missense mutation into the coding sequence of the hFIX. In parallel, the external forward and reverse primers (F9-HindII and F9-BamHI) were designed respectively for the $5^{\prime}$ and $3^{\prime}$ ends of the pre-pro-hFIX coding sequence. The F9-HindII forward primer was equipped with Hindll restriction site at the 5 ' end. The kozak sequence was also considered within the forward primer to ensure the efficient initiation of the translation of the cloned gene. The F9-BamHI reverse primer was equipped with BamHI restriction site at the 5 ' end. The $6 x \mathrm{His}$ codons was also considered at the $5^{\prime}$ end of the reverse primer before stop codon to introduce a $6 x$ His-tag to the C-terminus of the recombinant proteins. The amplification of the mutant coding sequence was performed using pfu DNA polymerase during which recombinant plasmid, pCDNA3-hFIXN ${ }^{\mathrm{N}}$, harboring coding sequence of the native hFIX, was used as a template. The amplified mutant sequence was cloned into 
pCDNA3.1 expression plasmid, within the Hindll and BamHI restriction sites downstream to the CMV promoter. After that, the prepared recombinant pCDNA3-hFIXM plasmid (expressing G4S-mutant hFIX) was transformed into $E$. coli DH5a competent cells for downstream verification using PCR and restriction enzyme digestion. The recombinant construct was further confirmed by DNA sanger sequencing.

Table 1: The primer sequences used in this study

\begin{tabular}{|ll|}
\hline Primer & Sequence $\left(\mathbf{5}^{\prime} \boldsymbol{\rightarrow} \mathbf{3}^{\prime}\right)$ \\
\hline G4S-F & AgAggTATAATTCAA_tAAATTggAAgAgTTTg \\
\hline G4S-R & ACAAACTCTTCCAATTTACITgAATTATACCTC \\
\hline F9-HindllI & CCCAAgCTTgCCACCATgCAgCgCgTgAAC \\
\hline F9-BamHI & CgCggATCCTCATTAATgATgATgATgATgATgAgTgAgCTTTgTTT TTTCC \\
\hline
\end{tabular}

\section{Cell Culture, transfection and transient protein expression}

Human embryonic kidney cells (HEK293T) was used as a host for transient expression of the both native and G4S-mutant hFIX. The HEK293T cells were cultured in 1:1 mixture of DMEM/F12 media (Gibco) supplemented with $10 \%$ fetal bovine serum (FBS) and $100 \mathrm{U} / \mathrm{ml}$ penicillin and $100 \mu \mathrm{g} / \mathrm{ml}$ streptomycin and incubated at $37^{\circ} \mathrm{C}$ under $5 \% \mathrm{CO}_{2}$ atmosphere.

Lipofactamine ${ }^{\circledR} 2000$ transfection reagent was used to transfect HEK293T cells with the recombinant plasmids expressing either the native or the mutant the $\mathrm{hFIX}$, according to the manufacturers procedure. In brief, $3.5 \mu \mathrm{g}$ plasmid DNA and $10 \mu$ Lipofactamin 2000 was separately diluted in $150 \mu$ serum free DMEM medium. The diluted DNA was mixed with the diluted reagent and incubated for 5 minutes at room temperature to allow DNA-lipid complex to be formed. The DNA-lipid complex was added to the HEK293T cells at the $60-70 \%$ confluence in a 6-well plate. In order to obtain biologically active recombinant $\mathrm{hFIX}$, vitamin $\mathrm{K}$ was added to the culture media at a final concentration of $2.5 \mu \mathrm{g} / \mathrm{ml}$, three to five hours after transfection.

\section{SDS-PAGE and western Blotting}

The native and G4S-mutant hFIX was expected to be secreted into the culture media due to the presence of the N-terminal signal peptide. Three days after transfection, the culture media were harvested and subjected for gradient SDS-PAGE and western blotting analysis using rabbit polyclonal anti-human coagulation factor IX. The gradient SDS-PAGE analysis was performed using $5 \%$ stacking and $4-12 \%$ resolving gel (Walker, 1984) to characterize the glycan attachment to the new and non-native $\mathrm{N}$ glycosylation site and the occurrence of hyperglycosylation. 
The electroblotting of the protein bands onto polyvinylidene difluoride (PVDF) membrane (Roche, Germany) was performed using wet procedure for three hours at $300 \mathrm{~mA}$ in a transfer buffer containing 25 mMTris, $192 \mathrm{mM}$ glycine and $20 \%$ methanol. The blot was then blocked for one hour using $5 \%$ non-fat milk in Tris-Buffered Saline containing 0.1 Tween 20 (TBST) and washed 3-5 times for 5 minutes each with TBST. Next, the blot was incubated with rabbit anti-hFIX polyclonal primary antibody (Abcan, UK) with a dilution of $1 / 2500$ in $3 \%(w / v)$ BSA in TBST. After one-hour incubation, the blot was again washed 3-5 times and incubated with a dilution of $1 / 2500$ goat anti-rabbit secondary antibody conjugated with peroxidase (Millipore, Co. US). Finally, the blot was washed thoroughly with TBST for 3-5 times and immunoreactive bands were visualized using enhanced chemiluminescence $(E C L)$ western blotting detection kit (Najm, Iran).

\section{Bioinformatics studies}

\section{Target sequence and template selection}

The primary sequence of the hFIX was obtained from the UniProt (accession no. P00740) and the first 45 residues from N-terminus of the mature hFIX, corresponding to the GLA domain of the hFIX, were chosen. The crystallographic structure of the calcium-stabilized GLA domain of the hFIX with resolution of $2.2 \mathrm{~A}^{\circ}$, was retrieved from RCSB protein databank (PDB ID: 1NL0) (Huang et al., 2004) and used as a template for homology-based modeling of the native and G4S-mutant GLA domains.

\section{Homology modeling and validation of the models}

The 3D structures of the native and G4S-mutant domains were predicted using MODELLER 9v10 software (Eswar et al., 2006) as described previously (Ghasemi et al., 2016). About 10,000 models were generated for each sequence and one model with the lowest value of the Probability Density Function (PDF) was chosen as the best final model. The stereochemical qualities of the final models were evaluated using PROCHECK (Laskowski et al., 1993), ProSA-web (Sippl, 1993; Wiederstein \& Sippl, 2007) and Qmean programs (Benkert et al., 2009) and compared to the template. Also the backbone RMSD values of the models relative to the template were calculated using the SPDBV program (Guex \& Peitsch, 1997). The verified final models were used as initial 3D structures for MD simulation.

\section{Molecular dynamics simulation}

Molecular dynamics (MD) simulation was performed by using GROMACS 5.4.1 package (Van Der Spoel et al., 2005) for studying conformational changes and stability conditions of the models in two different physiological conditions, in solution and membrane bound states. Therefore, topology files of the both native and mutant structure were separately prepared in two states, as follows: 


\section{Simulation of the peptides in solution}

In order to carry out MD simulation in solution, Amber ff99SB-ILDN force field parameters were utilized to describe the peptides. Moreover, the force field parameters for the Gla residue were generated using ANTECHAMBER with the general AMBER force field (gaff) (Wang et al., 2004). For simulation, the target peptide was placed within the center of a rectangular box with a distance of $10 \AA$ from all edge and solvated with TIP3P water model (Jorgensen et al., Klein, 1983).

The system was then neutralized by randomly placing $\mathrm{Na}^{+}$counter ions. Then, the whole system was subjected for 1000 steps of energy minimization and two steps of equilibration. At first, the entire system was equilibrated under constant volume and temperature conditions (NVT) for 500 ps. Afterward, 500 ps of equilibration was performed in the NPT ensemble to relax the system. Finally, a production run was performed for 80 ns under an NPT ensemble for sampling and analysis.

\section{Model building of the membrane}

For simulation of membrane-bound state, a patch of lipid bilayer including 144 molecules of 1,2dioleoylsn-glycero-3-[phospho-L-serine] (DOPS) was generated by Charmm Online Membrane Builder (http://www.charmm-gui.org) (Jo et al., 2008). Amber force field parameters were used to describe the DOPS. The resulting system was quadrupled in the XY plane. The area of the system was $51.55 \times$ $51.55 \AA^{2}$ which is large enough to accommodate the native or mutant GLA domains without altering its average density. Two slabs of water molecules were placed on each side of the lipid bilayer.

Subsequently, the solvated system was neutralized by placing $144 \mathrm{Na}^{+}$ions in the bulk water randomly. However, the assembled system consists of 2730 water molecule, $144 \mathrm{Na}^{+}$ions and 144 DOPS molecules (72 in each leaflet) in a $51.55 \times 51.55 \AA^{2} \times 113.17$ rectangular space.

The final system was then subjected to 5000 steps of energy minimization and five stages of equilibration. At first, the whole system was restrained by harmonics constraints at a constant temperature of $303 \mathrm{~K}$ for $125 \mathrm{ps}$. Next, the ions were set free to equilibrate at NVT for $125 \mathrm{ps}$. Then, the system was equilibrated at constant pressure and temperature (NPT) for 125 ps with restraint on the entire system except for ions. After that, the entire system, except the membrane, could move freely in the configuration space for $500 \mathrm{ps}$ in the NPT ensemble. At the next step, the harmonic constraint on the membrane was decreased and the system was equilibrated for another 500 ps. Finally, 1 ns production run was conducted at $303 \mathrm{~K}$ and 1 bar without any constraint. The equilibrated membrane was applied for modeling and simulation of the membrane-bound peptides. In order to simulate the membrane-bound native or GLA domains, at first, three adjacent DOPS molecules in the same leaflet of the lipid bilayer near the center of box were removed. Therefore, enough space was produced for insertion of the $\omega$-loop into the membrane. At the second step, the peptide was placed above the vacated space, with the $\omega$-loop pointing toward the membrane and slightly above the surface of the bilayer (Fig. 1). Next, some water molecules which overlapped with the peptide (closer than $3 \AA$ ) were removed. Then 1000 steps of energy minimization were performed on the obtained structure. After that, 500 ps equilibration in the NVT 
ensemble was performed on the membrane-bound GLA domains. All heavy atoms of the peptide were restrained with a force constant of $1000 \mathrm{~kJ} /\left(\mathrm{mol}_{\mathrm{nm}}{ }^{2}\right)$ to equilibrate the position of the lipid, water and ions. The system was then equilibrated at NPT for 500 ps with the same harmonic constraint. The final membrane-bound structures along with the solvent and ions were considered as the input of the simulation. A production run was carried out for $80 \mathrm{~ns}$ under an NPT ensemble for sampling and analysis.

\section{Simulation protocol}

it is necessary to mention that in the both simulation states, Lincs algorithm was used to constrain all bond involving hydrogen atoms (Hess et al., 1997). Electrostatic interactions were treated by using the particle Mesh Ewald (PME) method (Essmann et al., 1995). Periodic boundary conditions (PBC) were applied in the three-dimensional space. Leap-frog integration scheme was used with a $2 \mathrm{fs}$ time step. A cutoff distance was set to $10 \AA$ for van der Waals interactions. Temperature and pressure were controlled with Berendsen thermostat (Berendsen et al., 1984) and Parrinello-Rahman barostat (Parrinello \& Rahman, 1981) with a damping frequency of $0.1 \mathrm{ps}^{-1}$.

\section{Results And Discussion}

Missense mutations are the most prevalent mutation among hemophilia B patients (Goodeve, 2015; Melchiorre et al., Castaman, 2016; Melchiorre et al., 2016). These mutations may result in the creation of an extra N-glycosylation site through single amino acid substitution (Vogt et al., 2005; Vogt et al., 2007). Based on the literature and Factor IX Variants Database (http://www.factorix.org/), we found only one Nglycosylation mutation within GLA domain of the hFIX. This mutation which is caused by the nucleotide $\mathrm{G} / \mathrm{A}$ transition at position 148 of the hFIX coding sequence, results in the G4S amino acid substitution and creation of a new N-glycosylation site with $\mathrm{Asn}^{2}-\mathrm{Ser}^{3}-\mathrm{Ser}^{4}$ sequence in the GLA domain of the hFIX (Saad et al., 1994; VAN de Water et al., 1996). It is worth mentioning that mere presence of the $\mathrm{N}$ glycosylation site never guarantees oligosaccharide attachment to the Asn residue within the $\mathrm{N}$ glycosylation sequon (Lowenthal et al., 2016); therefore, G4S mutant was selected and then the glycan attachment to the non-native $\mathrm{N}$-glycosylation site was investigated.

\section{Experimental Analysis}

\section{Construction of the recombinant expression plasmids}

To clarify the glycan attaches to the new and non-native N-glycasylation site in the G4S mutant hFIX, in hemophilia B patients the nucleotide sequence encoding the native pre-pro-hFIX was amplified and the G4S mutation was then introduced into the coding sequence of the hFIX using overlap extension PCR method. The coding sequences of the native and mutant hFIX were separately cloned into the pcDNA3.1 expression plasmid downstream to the CMV promoter and transiently transfected into the HEK293 cells. 


\section{Immunblotting Of The Recombinant Native And G4s-mutant Hfix}

The oligosaccharide attachment to a protein results in an increase in the molecular weight of the glycoprotein that is detectable in a reducing gradient SDS-PAGE. To this aim, the HEK293T cells were transfected with recombinant plasmids expressing either native or G4S-mutant hFIX. Three days after transfection, the culture media were harvested and subjected for the gradient SDS-PAGE and western blotting analysis. The recombinant proteins were detectable with specific anti-hFIX polyclonal antibody. Both native and mutant proteins migrated the same distance and showed the same size in the gradient SDS-PAGE, showing no glycan attachment to the new N-glycosylation site of the G4S-mutant (Fig. 2).

By this result it can be concluded that the reduced clotting activity cannot be due to the glycan attachment to the G4S-mutant hFIX. The structural changes due to replacement of the Gly by Ser might directly influence the hFIX clotting activity. To understand the effects of the G4S mutation on the 3D structure of the hFIX GLA domain, bioinformatics studies and molecular dynamics simulations were conducted.

\section{Bioinformatics Analysis}

\section{The homology modeling of the G4S-mutant GLA domain and validation of the structure}

To evaluate the effects of the mutation on the 3D structure and dynamic behavior of the GLA domain, both native and G4S-mutant GLA domains were modeled using MODELLER 9v10 program in which 1NL0 chain $\mathrm{G}$, was used as a template. Among 10,000 generated models a structure with the lowest PDF value was chosen as a final best-model (Fig. 3).

The qualities of the models were then evaluated using PROCHECK, ProSA-web and Qmean tools. The Ramachandran plots of the both models were identical and the results indicated that $97.6 \%$ of the residues were presented within the most favored and additionally allowed regions and $2.4 \%$ of the residues were placed in the generously allowed region, while there was no amino acid within the disallowed region (Fig. 4A and B, Table 2). 
Table 2

Validation of the predicted 3D models for the native and G4S-mutant GLA domains using different scores.

\begin{tabular}{|llllllll|}
\hline Peptide & \multicolumn{2}{l}{ Ramachandran plot quality (\%) } & & $\begin{array}{l}\text { ProSA } \\
\text { Z-score }\end{array}$ & $\begin{array}{l}\text { Qmean } \\
\text { Z-score }\end{array}$ & $\begin{array}{l}\text { RMSD } \\
(\AA)\end{array}$ \\
\cline { 2 - 5 } & Most & Additionally & $\begin{array}{l}\text { Generously } \\
\text { allowed }\end{array}$ & Disallowed & & & \\
\hline favored & allowed & & & & & \\
\hline $\begin{array}{l}\text { G4S- } \\
\text { mutant }\end{array}$ & 87.8 & 9.8 & 2.4 & 0.0 & -3.86 & -0.42 & 0.27 \\
\hline
\end{tabular}

Also, the Z-scores of the both models were negative, -3.86 and -3.56 respectively, within the range of the scores typically found for native proteins of similar size (Table 2 and Fig. $4 \mathrm{C}$ and D). The qualities of the models were further validated by Qmean Z-score calculation

(Table 2).

As the final confirmation of the qualities of the models, the RMSD values of the models relative to the template (1NL0, chain G) were calculated (Table 2). The models had low RMSD values, indicating in a high similarity between the models and the template. The low RMSD values along with the abovementioned quality assessments supported the quality of the models and, led us to consider them as input files for the MD simulation.

\section{Molecular Dynamics Simulation}

MD simulation provides valuable atomistic insights into the structural and energetic factors influencing the interaction of peptide and membrane which cannot be collected by experimental procedure. To compare the dynamic behaviors of the mutant GLA domain with its native counterpart, and investigate the differences in their interactions with the membrane in solution, $80 \mathrm{~ns}$ MD simulation was conducted. At first, the both native and mutant peptides were simulated in water without membrane to understand the structural changes and extent of stability in solution as a control system. Then the both peptides were inserted in the DOPS bilayer membrane in solution.

\section{Stability analysis}

Root mean square deviations (RMSD) is a central criterion to understand the structural details, stability, flexibility and conformational behavior of proteins (Klepeis et al., 2009). In order to investigate effects of the G4S mutation on the overall stability of the GLA domain, the Ca RMSD values for the both native and mutant peptides against initial minimized structure were calculated in the both free and membranebound states, during the course of the simulation, and plotted as a function of time (Fig. 5). In the Fig. 5 it can be seen that the RMSD values of the native peptide in the free state was in the range of 1 to $2.3 \mathrm{~A}^{\circ}$, 
while the RMSD values for the free mutant GLA domain fluctuated between 1.5 to $2.8 \mathrm{~A}^{\circ}$. Moreover, RMSD values of the native and mutant peptides in membrane-bound states were in the range of 1 to 2.5 and 1.5 to $3 A^{\circ}$, respectively.

Based on the RMSD plots provided in Fig. 5, although the fluctuation patterns of the native and mutant peptides were almost similar in each state, the RMSD values of the native GLA domain were lower than those calculated for the mutant counterpart, both in free and membrane-bound states. Besides, the mutant GLA domain in free state showed a remarkably high peak in the middle of the simulation around $40 \mathrm{~ns}$ of the simulation time, which was absent in the native counterpart (Fig. 5A). As can be seen in Fig. 5, the RMAD values of the native and mutant peptides in membrane-bound state were higher than those in the free state. Moreover, the interaction of the peptide with the membrane resulted in the higher deviation in the structures of the both native and mutant peptides relative to their initial minimized structures, demonstrating the effects of the membrane on the both native and mutant structures (Fig. 5B). Some more details about the average RMSD values for the entire GLA domain (residues 1-45), $\omega$-loop (residues 1-11) and three helical regions of the native and mutant peptides in the both free and membrane-bound states are provided in Table 3. The results showed that the average RMSD values of the whole mutant GLA domain in the both states were higher than those calculated for the native counterparts (Table 3).

Table 3

Evaluation of the average RMSDs of different regions of the native and G4S-mutant GLA domains in the both free and membrane-bound states. F: free state, Mem: membrane-bound state.

\begin{tabular}{|lllll|}
\hline $\begin{array}{l}\text { Peptide name } \\
\text { Region name }\end{array}$ & F-native & F-mutant & Mem-native & Mem-mutant \\
\hline All & 1.41 & 1.78 & 2.06 & 2.40 \\
\hline Helix A & 0.16 & 0.20 & 0.25 & 0.27 \\
\hline Helix B & 0.25 & 0.28 & 0.38 & 0.57 \\
\hline Helix C & 0.54 & 0.70 & 0.62 & 1.01 \\
\hline w-loop & 0.47 & 0.55 & 0.93 & 1.23 \\
\hline
\end{tabular}

Previous studies have demonstrated that the interaction of the hFIX with the phospholipid bilayer is mediated by the residues 3-11 within the w-loop of the GLA domain (Cheung et al., 1992). The average RMSD values calculated for the $\omega$-loops of the native and mutant peptides in the membrane bound state were 0.93 and $1.23 \AA$ respectively, showing influence of the mutation on the structure of the $\omega$-loop. In the membrane bound state, the average RMSD values of the helices $C$ in the native and mutant GLA domain were 0.62 and 1.01 respectively, showing higher structural deviation in helix $\mathrm{C}$ of the mutant peptide in comparison with the same region in the native counterpart (Table 3). 
Radius of gyration $(\mathrm{Rg})$ is an indicator of the compactness of a protein structure and gives some information about the folding state of a protein (Lobanov et al., 2008). In the case of a stably-folded protein, $\mathrm{Rg}$ value remains relatively constant during a simulation time, while it changes in an unfolded protein. In order to characterize the compactness of the native and mutant GLA domains, in the both free and membrane bound states, the Rg values of each system were calculated during simulation time and plotted as a function of time (Fig. 6).

As depicted in Fig. 6, the fluctuations of the native structure were quite small, between 10.87 to $11 \AA$ in the both free and membrane-bound states, while the Rg values of the mutant peptide in the membranebound state displayed a sudden increase around 20 that continued until $60 \mathrm{~ns}$ of simulation. Therefore, the mutant GLA domain showed higher structural fluctuation in comparison with the native one.

\section{Flexibility profile analysis}

Root mean square fluctuation (RMSF) is another criterion to study the flexibility of the system. It indicates the mobility of a certain residue around its mean position. The RMSF values of the $\mathrm{Ca}$ atoms versus residue numbers were calculated to investigate the effects of the mutation on the residues fluctuations of the peptides in the both free and membrane bound states (Fig. 7). In free state, the RMSF profiles of the native and mutant peptides were almost the same; however, in membrane-bound state, the RMSF values of the mutant peptide displayed higher fluctuations for residues 10 to the end of the peptide. Therefore, the G4S substitution resulted in a significant increase in the flexibilities of the mutant peptide (Fig. 7).

\section{Distance between Center of mass of the peptides and the membranes}

In order to investigate the binding properties of the native and mutant GLA domains to the membrane, the distances between the center of mass (COM) of the peptides and the membrane were measured during the $80 \mathrm{~ns}$ of simulation and plotted versus time (Fig. 8).

According to the plot in Fig. 8, the native peptide displayed a more uniform trend in comparison with the mutant peptide, which indicated in the favorable interaction of the native peptide with DOPS membrane. In the case of the mutant GLA domain the distance varied during the course of the simulation. The mutant peptide began to move away from the membrane after $20 \mathrm{~ns}$ and then started to approach the membrane after $30 \mathrm{~ns}$ of the simulation. Afterward, the mutant peptide again began to move far from the membrane during the last $5 \mathrm{~ns}$ of simulation. Based on the graph in Fig. 8, the native GLA domain showed more stable interactions with the membrane than the mutant counterpart.

\section{Secondary Structure analysis}

Secondary structure plot demonstrates the structural changes of a protein during an MD run. In this study, the secondary structural changes of the native and mutant GLA domains were assessed by using the Dictionary of Protein Secondary Structure (DSSP) algorithm (Livesay et al., 2004). The analysis of DSSP calculates the relationship between internal motion and secondary structures, like helices, beta sheets, 
and loops during simulation time. The results from DSSP analysis proposed that the mutant peptide underwent distinct conformational changes during the simulation whereas such drifts were not detected in the native peptide (Fig. 9). Moreover, all those conformational changes were in concordance with the time dependent structural fluctuations. According to the Fig. 9, the residues 15-19 and 25-31 (Helix A and $B$ respectively) of the mutant peptide in free state underwent transitions from a-helix towards bend structures, the structural changes that were not observed in the native peptide. Besides, the mutant peptide in the membrane-bound state tolerated much more conformational drifts than in the free state. All the data were closely in accordance with the results from RMSF analysis that showed higher flexibility for membrane-bound mutant GLA domain (Fig. 7). It means that binding of the mutant peptide to the membrane changes its secondary structure, while such deviations were not occurred for the native structure.

\section{Interaction energy and hydrogen bond analysis}

The contribution of electrostatic and van der Waals interactions between peptides and membrane were determined during the last $50 \mathrm{~ns}$ of the simulation (Table 4). It was found that native peptide binds to the membrane more strongly than the mutant peptide and forms more favorable interactions.

Table 4

Evaluation of energy contributions and the number of hydrogen bonds formed between amino acid side chains of a peptide (Peptide-Peptide), amino acid side chains and the membrane (Peptide-Membrane), or amino acid side chains and solvent (Peptide-Water). ELE: electrostatic energy, VDW: van der Waals energy.

\begin{tabular}{|llllll|}
\hline \multirow{2}{*}{ ELE } & VDW & \multicolumn{2}{l|}{ Hydrogen Bond } & \\
\cline { 4 - 6 } & & & Peptide-Peptide & Peptide-Membrane & Peptide-Water \\
\hline Mem-Nat & -250.16 & -207.39 & 39.65 & 13.74 & 140.51 \\
\hline Mem-Mut & -131.72 & -148.17 & 34.83 & 11.04 & 147.43 \\
\hline
\end{tabular}

Besides, the number of the hydrogen bonds were computed and averaged during the last $50 \mathrm{~ns}$ of the simulation time and presented in Table 4. For hydrogen bond analysis, a hydrogen bond was defined by a certain criterion: donor-acceptor distance $<3.5 \AA$ and donor-hydrogen-acceptor angle $>135^{\circ}$. Three kinds of hydrogen bonds were considered; the number of hydrogen bonds formed between amino acid side chains of a peptide (peptide-peptide), between the amino acid side chains, and the membrane (peptidemembrane), or amino acid side chains and water molecules (peptide-water). The average number of hydrogen bonds formed in peptide-peptide and peptide-membrane for the native peptide were greater than those for the mutant peptide. Likewise, the orientation of amino acids of the native peptide in the membrane led to the formation of the much more number of the hydrogen bonds between the peptide and the membrane. Therefore, the formation of more hydrogen bonds stabilized the binding of the native peptide to the membrane. 
Two snapshots of the native and mutant peptides bound to the membrane at 5 and 80 ns were shown in Fig. 10. The position and fluctuation of the structurally bound $\mathrm{Ca}^{2+}$ ions were not affected during the simulation (Fig. 10). The $\mathrm{Ca}^{2+}-6$ was in contact with helix1 (H1), while other $\mathrm{Ca}^{2+}$ ions were placed between peptide and DOPS molecules.

For understanding the role of $\mathrm{Ca}^{2+}$ ions, the atomic density profiles of the $\mathrm{Ca}^{2+}$ ions for the native and mutant peptide were calculated during the last $50 \mathrm{~ns}$ of the simulation (Fig. 11A) and compared to the density of DOP and $\mathrm{PO}_{4}{ }^{-}$. The atomic density profiles of $\mathrm{Ca}^{2+}$ ions displayed two peaks for the both native and mutant peptides; a shorter peak was related to the $\mathrm{Ca}^{2+}-6$ which positioned in longer distance relative to the membrane and was in contact with Gla15 and Gla20 (Fig. 11B) and a higher peak was related to the other $\mathrm{Ca}^{2+}$ ions and placed near the membrane.

The interaction between the negatively charged GLA domain and anionic DOPS was expected to be primarily mediated by the bound $\mathrm{Ca}^{2+}$ ions. As shown in Fig. $10, \mathrm{Ca}^{2+}$ ions are in contact with three DOPS molecules, and serve as anchors that help the peptide to stay on the surface of the membrane that means stable electrostatic interactions are formed between peptide, ions and membrane.

\section{Conclusion}

Our results from site-directed mutagenesis and the expression of the recombinant native and G4S-mutant hFIX in HEK293T cells showed no glycan attachment to the Asn residue located within the new Nglycosylation site. Moreover, molecular dynamics simulation of the native and mutant GLA domain demonstrated the partial structural changes for mutant peptide. Analysis of the RMSF profiles of the native and mutant GLA domains, indicated in the higher fluctuations and flexibilities for the amino acid residues of the mutant peptide in membrane-bound state. Besides, our results demonstrated that the native peptide binds to the membrane through more hydrogen bonds and forms more favorable van der Waals and electrostatic interaction compared to the mutant peptide. The increased flexibility and weak interactions of the mutant with the membrane, might explain the decreased coagulation activity of the G4S-mutant hFIX in hemophilia B patients.

\section{Declarations}

\section{Acknowledgment}

The author would like to thank National Institute of Genetic Engineering and Biotechnology of Iran for its facilities that made this research possible.

\section{References}

1. Autin L, Miteva M, Lee W, Mertens K, Radtke KP, Villoutreix B (2005) Molecular models of the procoagulant factor VIIla-factor IXa complex. Haemostasis 3:2044-2056 
2. Benkert P, Künzli M, Schwede T (2009) QMEAN server for protein model quality estimation. Nucleic acids research 37: (suppl_2), W510-W514

3. Berendsen HJ, Postma J, van Gunsteren WF, DiNola A, Haak JR (1984) Molecular dynamics with coupling to an external bath. J Chem Phys 81:3684-3690

4. Bolt G, Bjelke J, Hermit M, Hansen L, Karpf D, Kristensen C (2012) Hyperglycosylation prolongs the circulation of coagulation factor IX. Haemostasis 10:2397-2398

5. Casanova JL, Abel L (2007) Human genetics of infectious diseases: a unified theory. EMBO J 26:915-922

6. Cheung W, Hamaguchi N, Smith K, Stafford D (1992) The binding of human factor IX to endothelial cells is mediated by residues 3-11. J Biol Chem 267:20529-20531

7. Essmann U, Perera L, Berkowitz ML, Darden T, Lee H, Pedersen LG (1995) A smooth particle mesh Ewald method. J T J o c. p. (103(19):8577-8593

8. Eswar N, Webb B, Marti-Renom MA, Madhusudhan M, Eramian D, Shen M, Sali A J. C. p. i. b. (2006). Comparative protein structure modeling using Modeller. 15(1), 5.6. 1-5.6. 30

9. Ghasemi F, Zomorodipour A, Karkhane AA, Khorramizadeh MRJ, Modelling (2016) In silico designing of hyper-glycosylated analogs for the human coagulation factor IX. 68, 39-47

10. Gil G-C, Velander WH, Van Cott KEJG (2008) Analysis of the N-glycans of recombinant human Factor IX purified from transgenic pig milk. 18:526-5397

11. Goodeve ACJJ, O. T, Haemostasis (2015) Hemophilia B: molecular pathogenesis and mutation analysis 13(7):1184-1195

12. Guex N, Peitsch MCJ (1997) e. SWISS-MODEL and the Swiss-Pdb Viewer: annvironment for comparative protein modeling. 18(15), 2714-2723

13. Gui T, Lin H-F, Jin D-Y, Hoffman M, Straight DL, Roberts HR, Stafford DWJB (2002) Circulating and binding characteristics of wild-type factor IX and certain Gla domain mutants in vivo. The Journal of the American Society of Hematology 100(1):153-158

14. Hess B, Bekker H, Berendsen HJ, Fraaije JG (1997) J. J. o. c. c. LINCS: a linear constraint solver for molecular simulations. 18(12), 1463-1472

15. Huang M, Furie BC, Furie BJ (2004) J. o. B. C. Crystal structure of the calcium-stabilized human factor IX Gla domain bound to a conformation-specific anti-factor IX antibody. 279(14), 14338-14346

16. Jo S, Kim T, lyer VG, Im W (2008) J. J. o. c. c. CHARMM-GUI: a web-based graphical user interface for CHARMM. 29(11), 1859-1865

17. Jorgensen WL, Chandrasekhar J, Madura JD, Impey RW, Klein ML (1983) Comparison of simple potential functions for simulating liquid water. J T J o c. p. (79(2):926-935

18. Klepeis JL, Lindorff-Larsen K, Dror RO, Shaw DE (2009) Long-timescale molecular dynamics simulations of protein structure and function. Curr Opin Struct Biol 19(2):120-127

19. Laskowski RA, MacArthur MW, Moss DS, Thornton JM (1993) J. J. o. a. c. PROCHECK: a program to check the stereochemical quality of protein structures. 26(2), 283-291 
20. Livesay D, Dallakyan S, Wood G, Jacobs DJF (2004) I. A flexible approach for understanding protein stability. 576(3), 468-476

21. Lobanov MY, Bogatyreva N, Galzitskaya OJMB (2008) Radius of gyration as an indicator of protein structure compactness.. 42:623-6284

22. Lowenthal MS, Davis KS, Formolo T, Kilpatrick LE, Phinney KW (2016) Identification of novel Nglycosylation sites at noncanonical protein consensus motifs. J Proteome Res 15(7):2087-2101

23. Melchiorre D, Linari S, Castaman $G$ (2016) The higher prevalence of missense mutations in hemophilia B compared to hemophilia A could be important in determining a milder clinical phenotype in patients with severe hemophilia B. Haematologica 101(10):e429-e429

24. Melchiorre D, Linari S, Manetti M, Romano E, Sofi F, Matucci-Cerinic M, Castaman G (2016) Clinical, instrumental, serological and histological findings suggest that hemophilia B may be less severe than hemophilia A. Haematologica 101(2):219

25. Ohkubo YZ, Tajkhorshid EJS (2008) Distinct structural and adhesive roles of Ca2+ in membrane binding of blood coagulation factors.. 16:72-811

26. Parrinello M, Rahman AJJ (1981) o. A. p. Polymorphic transitions in single crystals: A new molecular dynamics method. 52(12), 7182-7190

27. Pegg CL, Zacchi LF, Recinos DR, Howard CB, Schulz BL (2020) Identification of novel glycosylation events on human serum-derived Factor IX. Glycoconj J 37(4):471-483

28. Persson KE, Astermark J, Björk I, Stenflo JJFI (1998) Calcium binding to the first EGF-like module of human factor IX in a recombinant fragment containing residues 1-85: Mutations V46E and Q50E each manifest a negligible increase in calcium affinity. 421(2),100-104

29. Saad S, Rowley G, Tagliavacca L, Green P, Giannelli F (1994) First report on UK database of haemophilia B mutations and pedigrees. Thromb Haemost 71(05):563-570

30. Sippl MJJPS, Function, Bioinformatics (1993) Recognition of errors in three-dimensional structures of proteins.. 17:355-3624

31. Vadivel K, Bajaj SP (2012) J. F. i. b. a. j., \& library, v. Structural biology of factor VIla/tissue factor initiated coagulation. 17, 2476

32. VAN DE WATER NS, BROWETT PJ (1996) Factor IX gene mutations in haemophilia B: a New Zealand population-based study. Haemophilia 2(1):24-27

33. Van Der Spoel D, Lindahl E, Hess B, Groenhof G, Mark AE, Berendsen H (2005) GROMACS: fast, flexible, and free. J J J o c c 26(16):1701-1718

34. Venkateswarlu DJB (2014) \& communications, b. r. Structural insights into the interaction of blood coagulation co-factor VIIla with factor IXa: A computational protein-protein docking and molecular dynamics refinement study. 452(3), 408-414

35. Vogt G, Chapgier A, Yang K, Chuzhanova N, Feinberg J, Fieschi C, BustamanteJ. J. N. g. (2005). Gains of glycosylation comprise an unexpectedly large group of pathogenic mutations.37(7),692700 
36. Vogt G, Vogt B, Chuzhanova N, Julenius K, Cooper DN, Casanova J-L (2007) Gain-of-glycosylation mutations. Curr Opin Genet Dev 17(3):245-251

37. Walker JM (1984) Gradient SDS polyacrylamide gel electrophoresis. Proteins. Springer, pp 57-61

38. Wang J, Wolf RM, Caldwell JW, Kollman PA, Case DA (2004) Development and testing of a general amber force field. J J o c c 25(9):1157-1174

39. Wei W, Misra S, Cannon MV, Yang R, Zhu X, Gilmore R, Zhang BJBJ (2018) Molecular mechanisms of missense mutations that generate ectopic N-glycosylation sites in coagulation factor VIII. 475:8738865

40. Wei W, Zheng C, Zhu M, Zhu X, Yang R, Misra S, Zhang BJS (2017) r. Missense mutations near the Nglycosylation site of the $\mathrm{A} 2$ domain lead to various intracellular trafficking defects in coagulation factor VIII. 7, 45033

41. Wiederstein M, Sippl MJ (2007) J. N. a. r. ProSA-web: interactive web service for the recognition of errors in three-dimensional structures of proteins. 35(suppl_2), W407-W410

42. Yoshitake S, Schach BG, Foster DC, Davie EW, Kurachi K (1985) Complete nucleotide sequences of the gene for human factor IX (antihemophilic factor B). Biochemistry 24(14):3736-3750

43. Zacchi LF, Roche-Recinos D, Pegg CL, Phung TK, Napoli M, Aitken C, Schulz BL (2021) Coagulation factor IX analysis in bioreactor cell culture supernatant predicts quality of the purified product.

Communications Biology 4(1):1-19

\section{Figures}




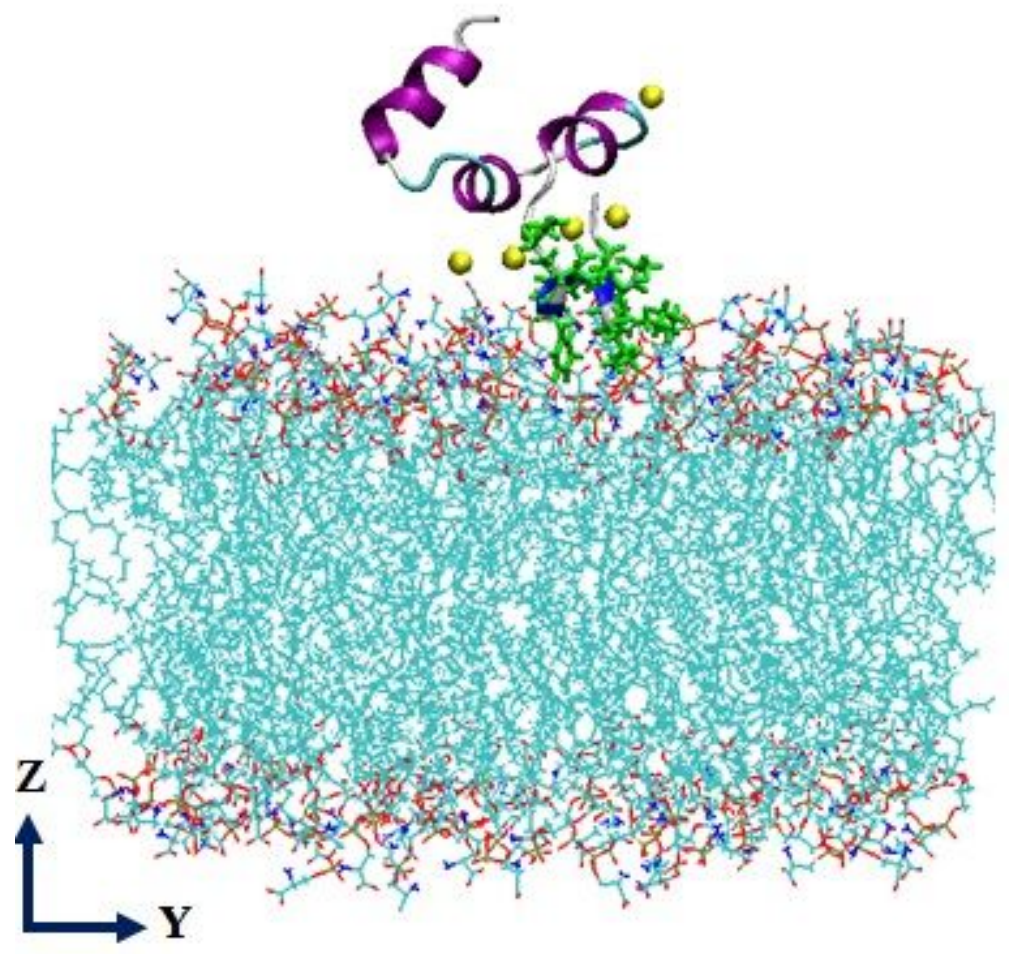

Figure 1

The schematic representation of the native GLA domain in interaction with DOPS membrane; the native GLA domain is represented by cartoon (purple), the bound $\mathrm{Ca} 2+$ ions are displayed as yellow circles, the DOPS molecules are shown with thin lines and the $\omega$-loop is represented by green surface.

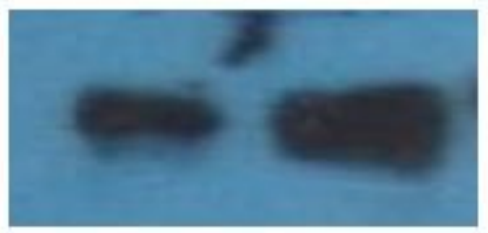

\section{Figure 2}

Western blotting analysis of the native and G4S-mutant hFIX transiently expressed by transfected HEK293T cells. Lane 1: native hFIX, Lane 2: G4S-mutant hFIX. 


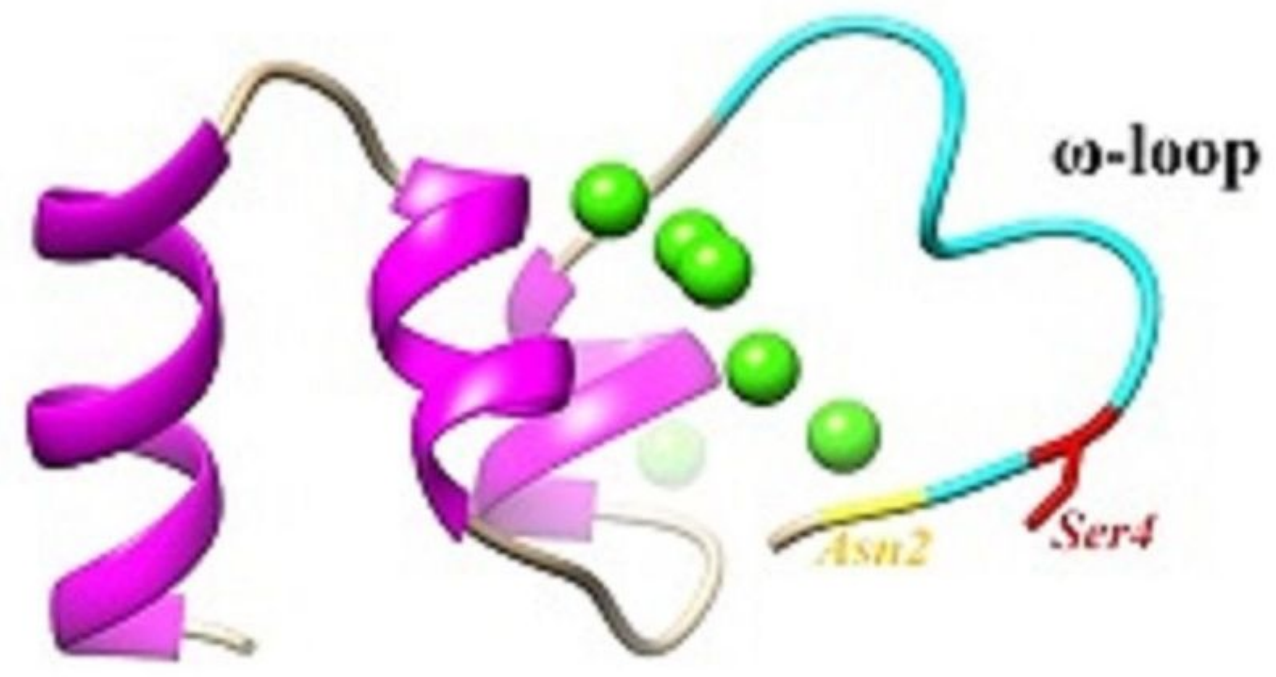

Figure 3

Cartoon representation of the modeled G4S-mutant GLA domain; the Ser4 residue is shown as red color, the $\mathrm{Ca} 2+$ ions are displayed as green spheres. 

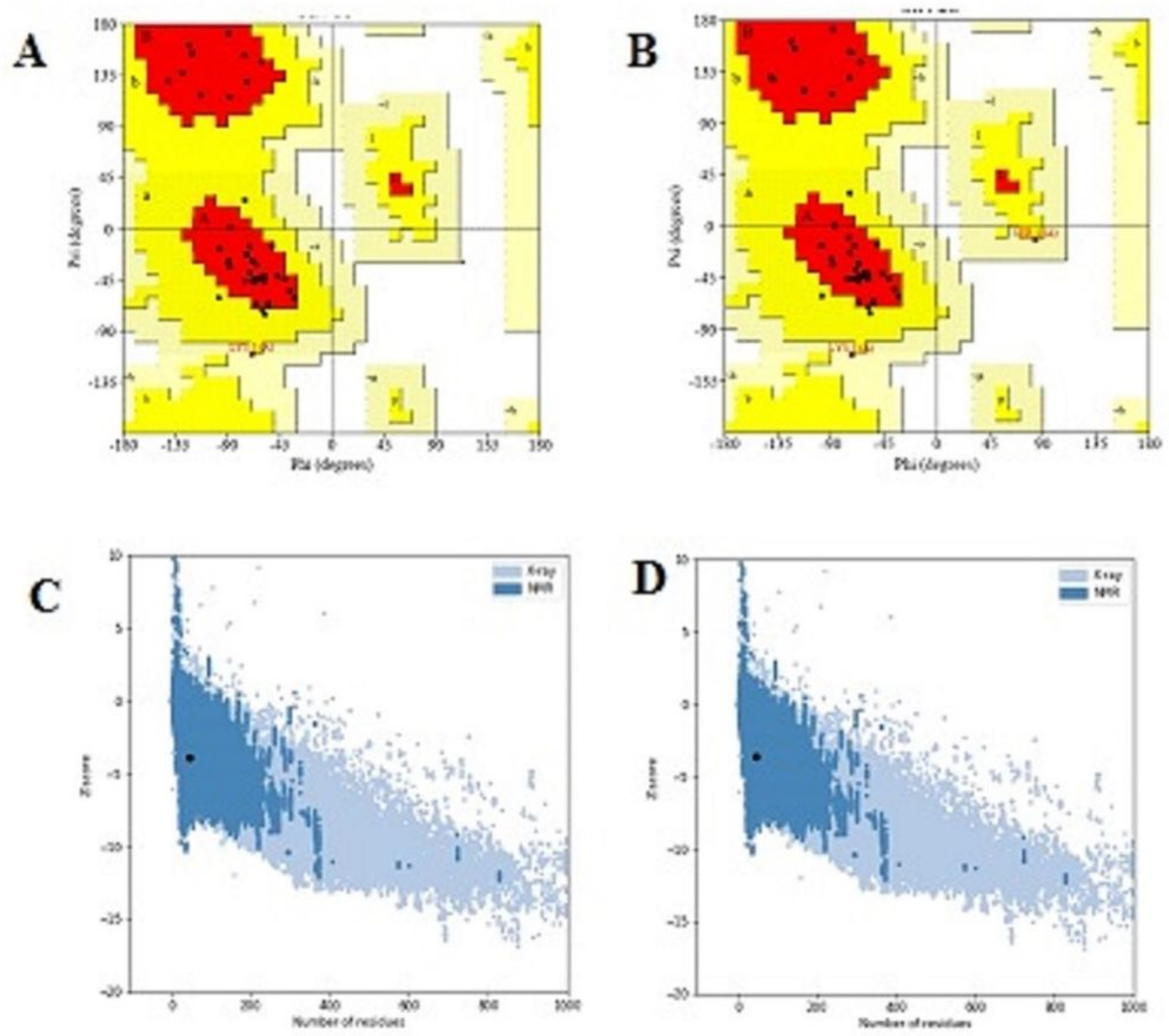

\section{Figure 4}

Validation of modeled native and G4S-mutant GLA domains. Ramachandran plots for the native (A) and G4S-mutant peptides (B). The most favored, additionally allowed, generously allowed and disallowed regions are shown in red, yellow, gray and white colors, respectively. ProSA Z-score plots for the modeled native (C) and mutant (D) GLA domains. 

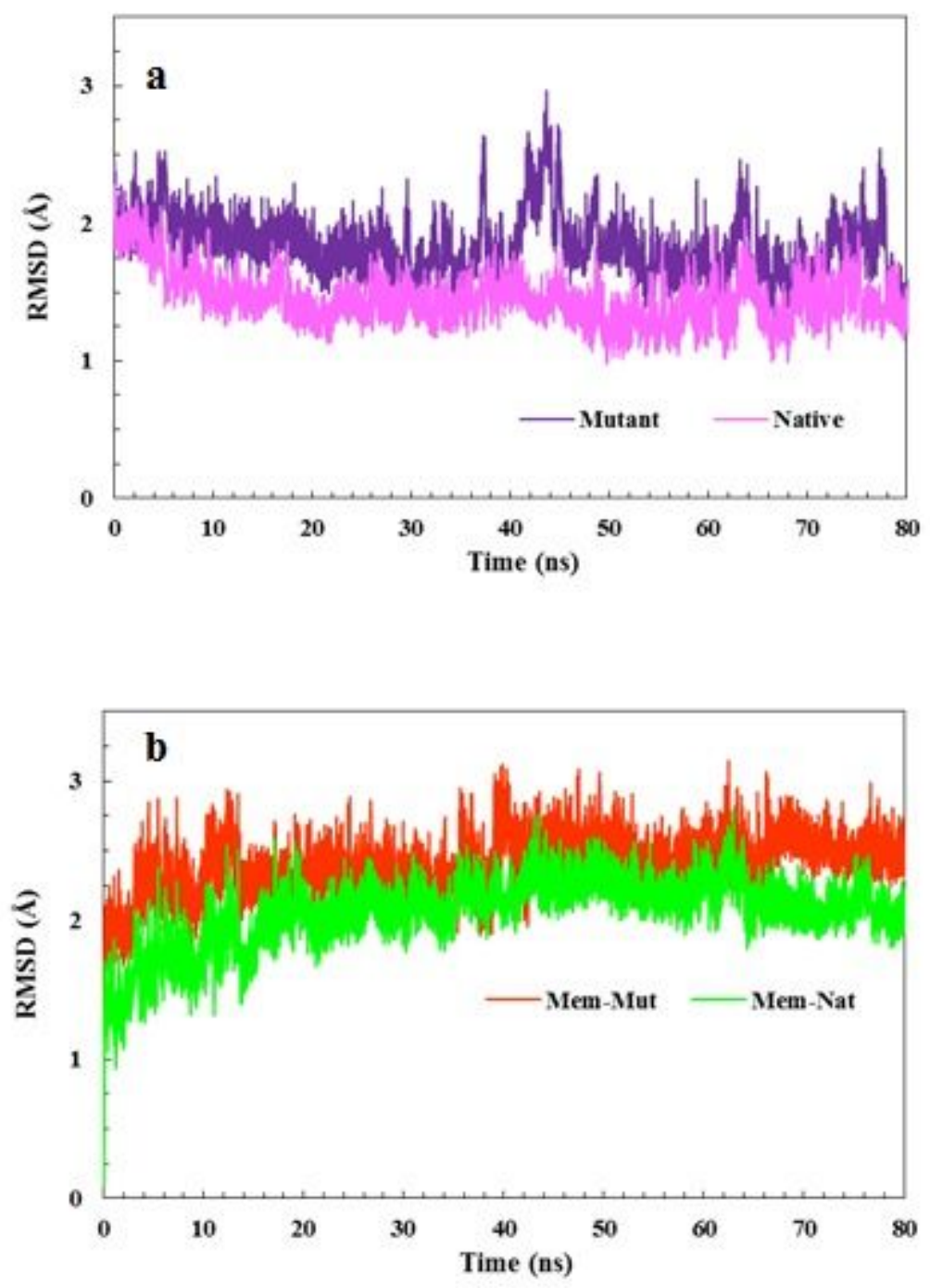

Figure 5

The RMSD plots of the $\mathrm{Ca}$ atoms relative to their initial immunized structure as a function of time. The native and G4S-mutant GLA domains in the solution (free) (A) and the membrane-bound (B) states are shown in magenta, purple, green, and red respectively. 

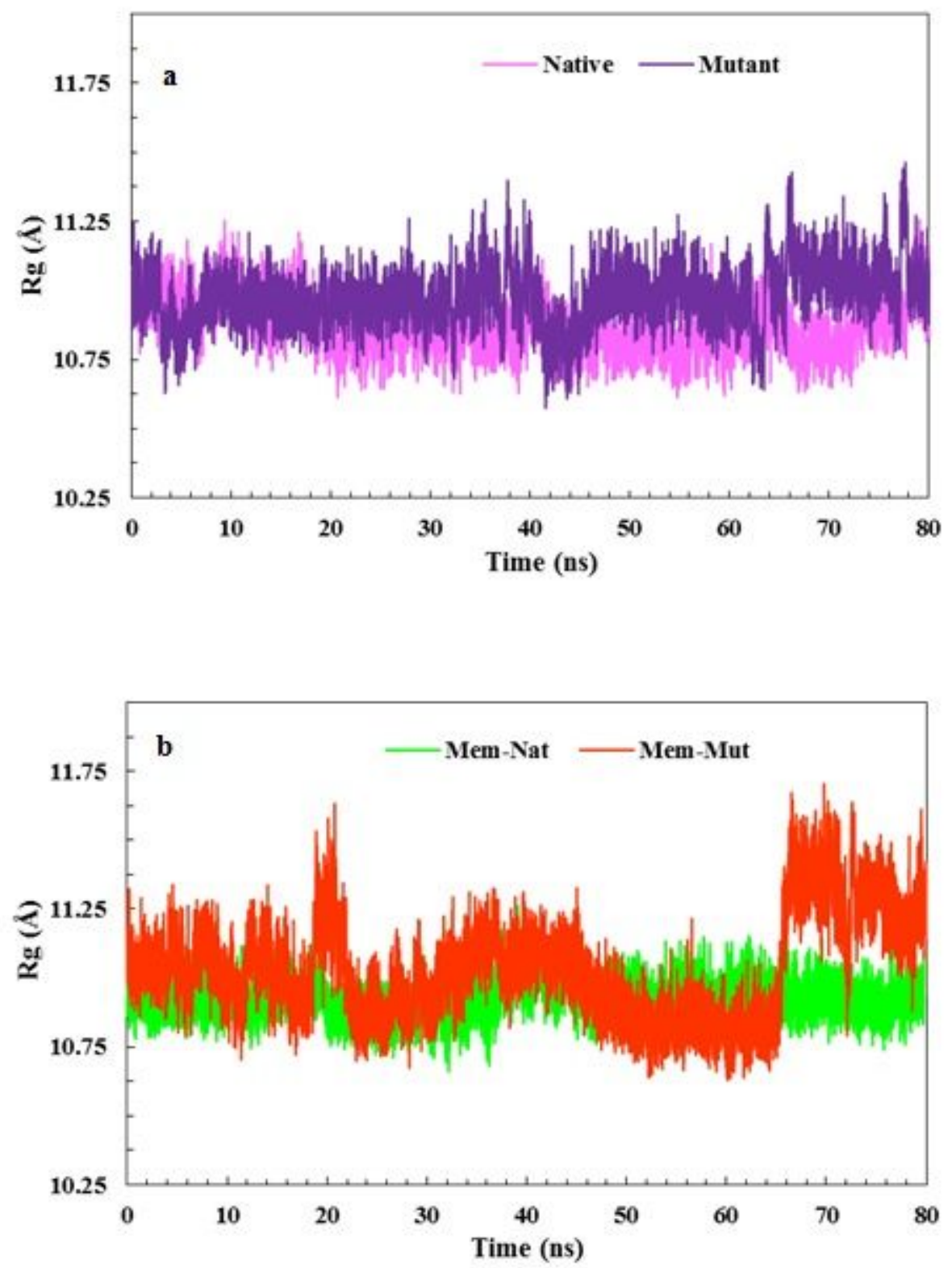

Figure 6

Radius of gyration $(\mathrm{Rg})$ plots of the peptides versus time. The native and G4S-mutant GLA domains in solution (free) (A), and the membrane-bound (B) states are shown in magenta, purple, green, and red respectively. 

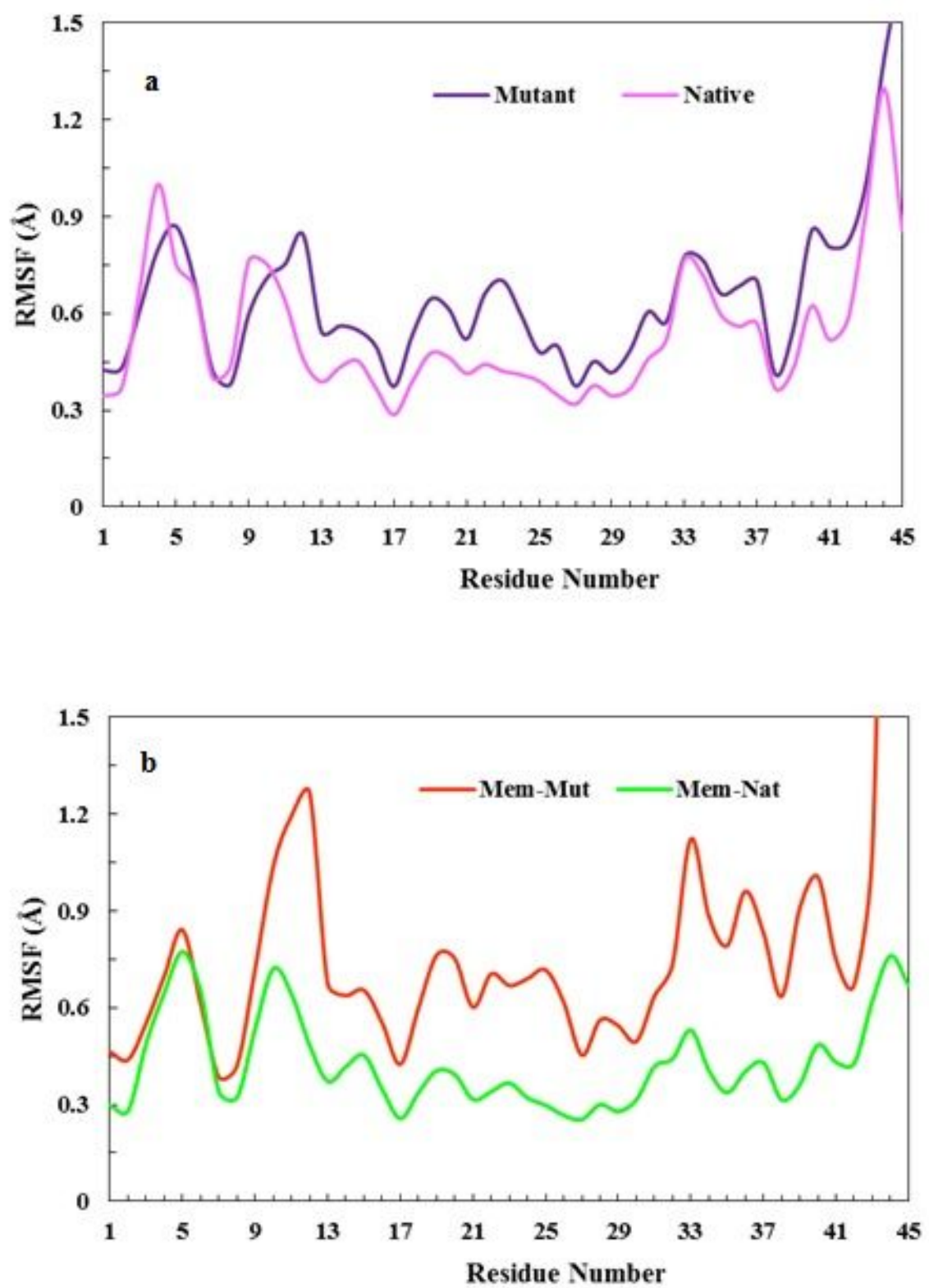

Figure 7

Analysis of Root mean square fluctuations (RMSFs) of the backbone Ca atoms of the peptides in the both free and membrane bound states. The native and mutant peptides in the solution (free) and the membrane-bound states are shown in magenta, purple, green, and red respectively. 


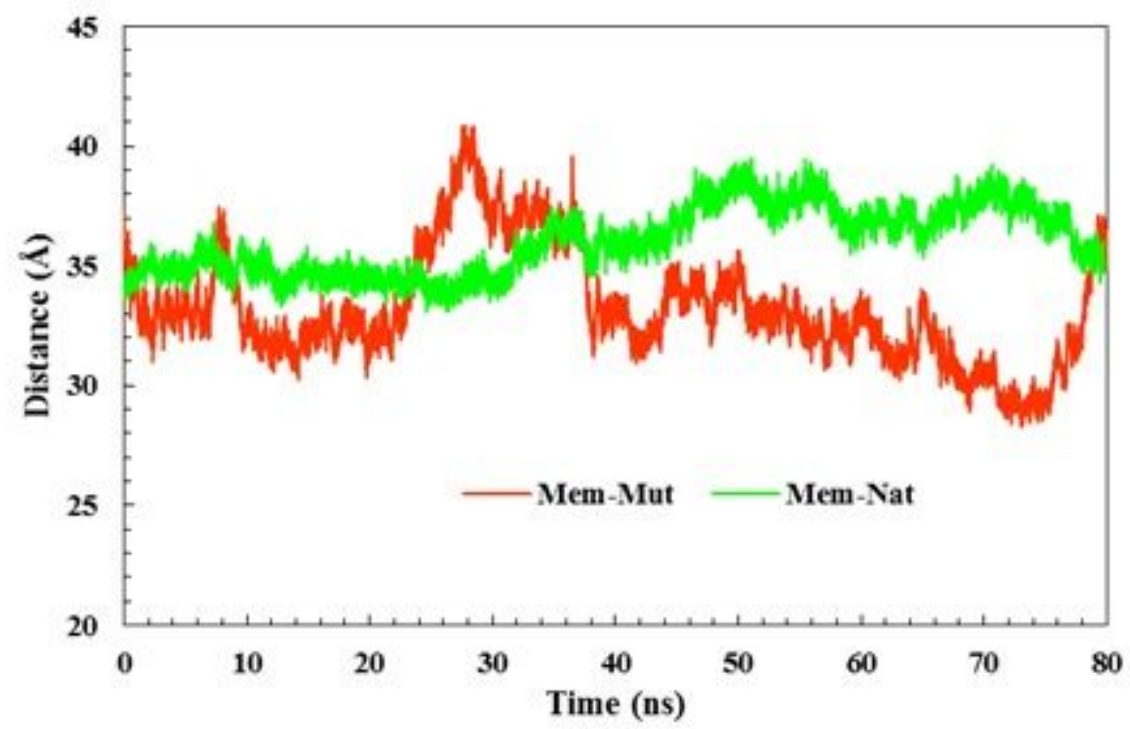

Figure 8

The Distance between center of mass (COM) of the peptides and the membrane as a function of time. The native and mutant peptides in solution (free) and membrane-bound states are shown in magenta, purple, green, and red respectively. 

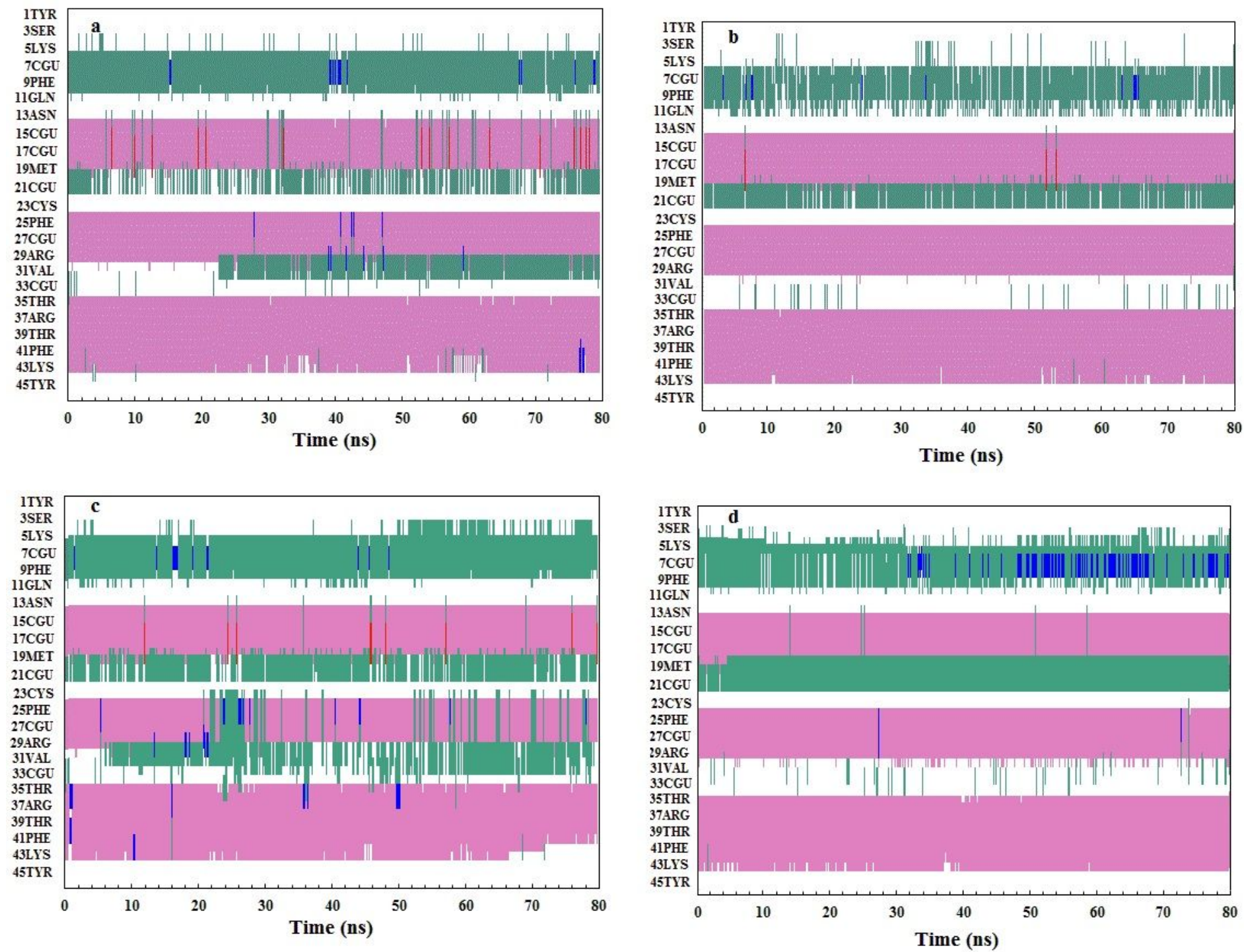

Figure 9

Evolution of secondary structures from DSSP analysis for the native and mutant GLA domains as a function of time. The secondary structure diagram of (A) the native GLA domain (free state), (B) the G4Smutant GLA domain (free state), (C) the native GLA domain (membrane-bound state), and (D) the G4S mutant GLA domain (membrane-bound state) as a function of time. T: Turn, E: Extended conformation, B: Isolated bridge, $\mathrm{H}$ : a-helix, G: 3-10 helix, I: Pi-helix, C: Coil. 

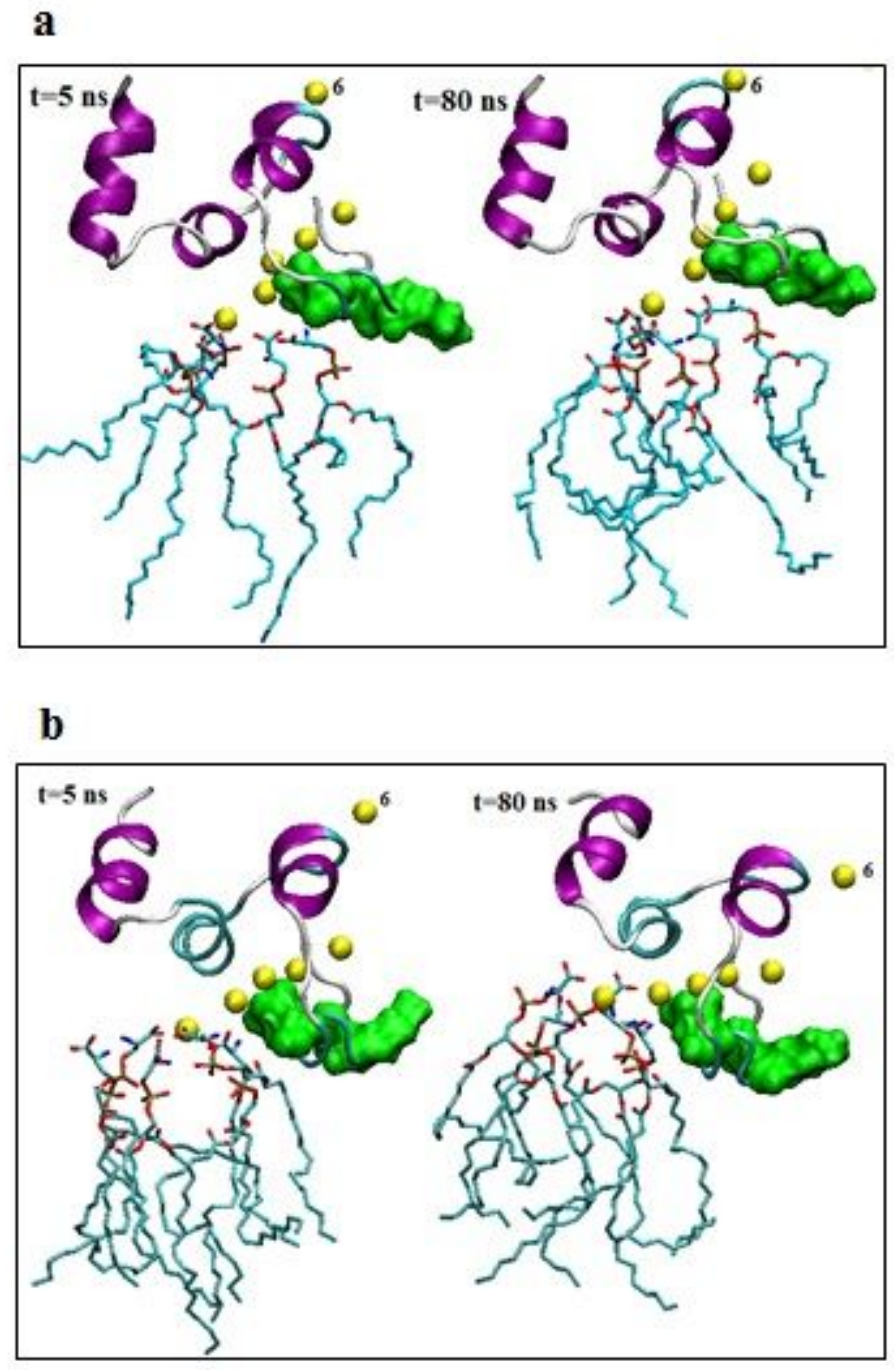

Figure 10

Evaluation of the membrane-bound native and mutant peptides. (A) snapshots of the native and (B) mutant peptides bound to the membrane. For better visualization, DOPS molecules are within $5 \AA$ of any $\mathrm{Ca} 2+$ ions (yellow sphere) are drawn with a licoric representation; $\omega$-loop are displayed by green surface. 

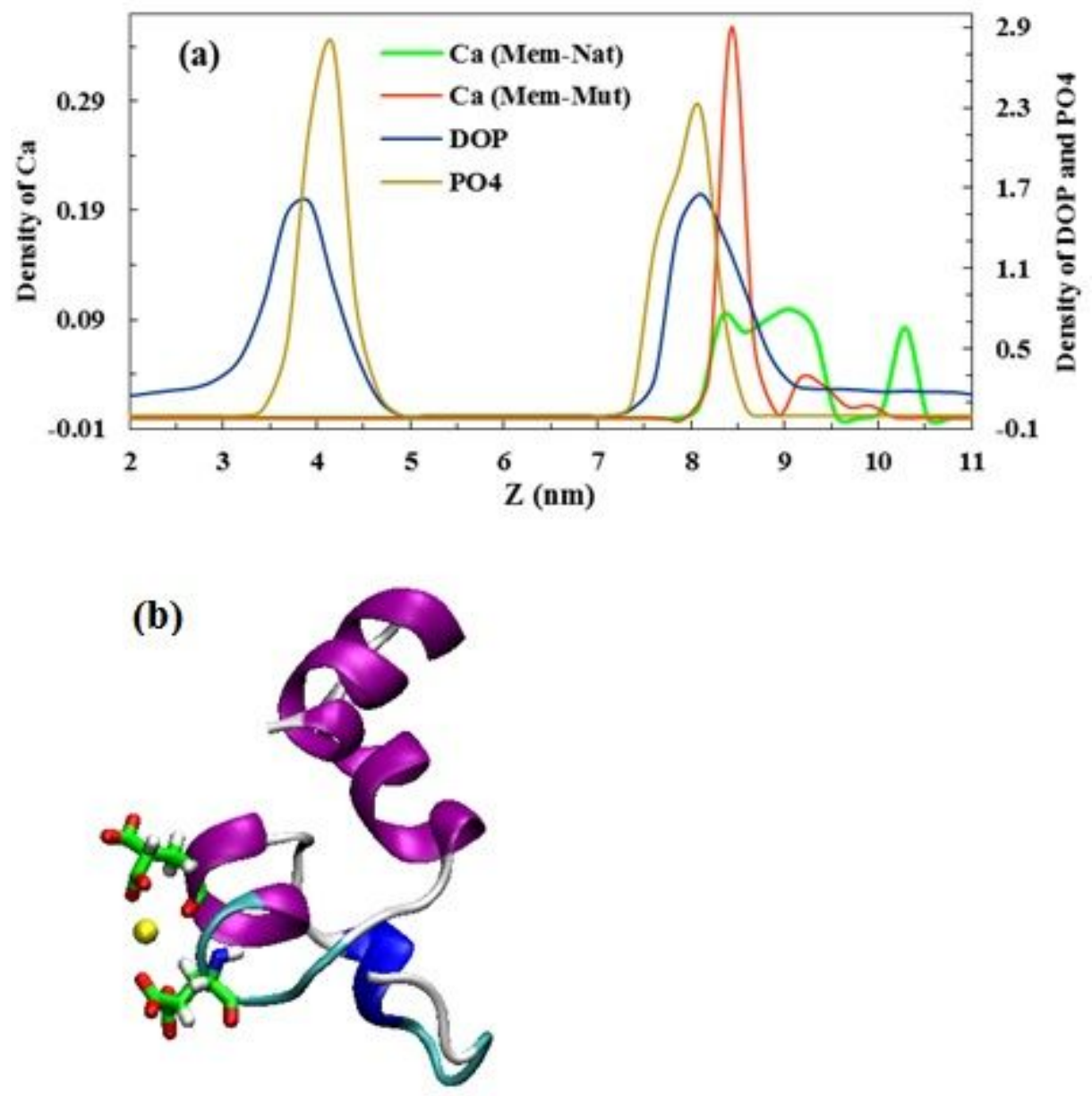

\section{Figure 11}

Analysis of atomic density profile for $\mathrm{Ca} 2+$ ions. (A) the atomic density plot of $\mathrm{Ca} 2+$ ions in the native and mutant peptides, DOPS membrane and P04-group. (B) The representation of Ca2+-6 ion which is in contact with Gla15 and Gla 20. 